\title{
An Algebraic Operator Approach to the Analysis of Gerber-Shiu Functions
}

\author{
Hansjörg Albrecher ${ }^{a}$, Corina Constantinescu ${ }^{b}$, Gottlieb Pirsic ${ }^{b}$, \\ Georg Regensburger ${ }^{b}$, Markus Rosenkranz ${ }^{b}$ \\ ${ }^{a}$ Institute of Actuarial Science, Faculty HEC, University of Lausanne, \\ Extranef Building, CH-1015 Lausanne, Switzerland. \\ ${ }^{b}$ Johann Radon Institute for Computational and Applied Mathematics (RICAM), \\ Altenbergerstrasse 69, A-4040 Linz, Austria.
}

\begin{abstract}
We introduce an algebraic operator framework to study discounted penalty functions in renewal risk models. For inter-arrival and claim size distributions with rational Laplace transform, the usual integral equation is transformed into a boundary value problem, which is solved by symbolic techniques. The factorization of the differential operator can be lifted to the level of boundary value problems, amounting to iteratively solving first-order problems. This leads to an explicit expression for the Gerber-Shiu function in terms of the penalty function.
\end{abstract}

\section{Introduction}

We consider the collective renewal risk model introduced by Sparre Andersen (1957) that describes the amount of free capital $U(t)$ at time $t$ in an insurance portfolio by

$$
U(t)=u+c t-\sum_{k=1}^{N(t)} X_{k} .
$$

Here $N(t)$ is a renewal process that counts the number of claims incurred during the time interval $(0, t]$, the constant $c$ is the premium rate and the random variables $\left(X_{k}\right)_{k \geq 0}$ denote the claim sizes that occur at random times

Email addresses: hansjoerg.albrecher@unil.ch, corina.constantinescu@oeaw.ac.at, gpirsic@gmail.com, georg.regensburger@oeaw.ac.at, markus.rosenkranz@oeaw.ac.at (Hansjörg Albrecher ${ }^{a}$, Corina Constantinescu ${ }^{b}$, Gottlieb Pirsic ${ }^{b}$, Georg Regensburger ${ }^{b}$, Markus Rosenkranz ${ }^{b}$ ) 
$\left(T_{k}\right)_{k \geq 0}$, with $\tau_{k}=T_{k}-T_{k-1}$ i.i.d. random variables denoting the $k$-th interclaim (or inter-arrival) time $\left(T_{0}=0\right)$. The initial surplus (after the claim at time 0 is paid) is given by $u \geq 0$. Moreover, $\left(X_{k}\right)_{k \geq 0}$ and $\left(\tau_{k}\right)_{k \geq 1}$ are assumed to be independent. Ruin occurs when the surplus process becomes negative for the first time, so the time of ruin is given by

$$
T_{u}=\inf \{t \mid U(t)<0\}
$$

and the ruin probability of a company having initial capital $u$ is given by

$$
\psi(u)=P\left(T_{u}<\infty \mid U(0)=u\right) .
$$

The net profit condition $c \mathbb{E}\left(T_{k}\right)>\mathbb{E}\left(X_{k}\right)$ is imposed to ensure that $\psi(u)<1$ for all $u \geq 0$.

Denoting by $f(x, y, t \mid u)$ the joint probability density function of the surplus immediately before ruin $U\left(T_{u}-\right)$, the deficit at ruin $\left|U\left(T_{u}\right)\right|$ and the time of ruin $T_{u}$, we have

$$
\int_{0}^{\infty} \int_{0}^{\infty} \int_{0}^{\infty} f(x, y, t \mid u) d x d y d t=\psi(u)
$$

Let $w(x, y)$ be a penalty function, nonnegative for $x \geq 0, y \geq 0$. Then for $u \geq 0$, the expected discounted penalty function (also called Gerber-Shiu function) is defined by

$$
\begin{aligned}
m(u) & =\mathbb{E}\left(e^{-\delta T_{u}} w\left(U\left(T_{u}-\right),\left|U\left(T_{u}\right)\right|\right) 1_{T_{u}<\infty} \mid U(0)=u\right) \\
& =\int_{0}^{\infty} \int_{0}^{\infty} \int_{0}^{\infty} e^{-\delta t} w(x, y) f(x, y, t \mid u) d x d y d t
\end{aligned}
$$

where $\delta>0$ is a discount rate.

Since the introduction of this function in the compound Poisson model in the papers of Gerber and Shiu (1997, 1998), there has been a vast literature on its analysis and extensions to more general models. Li and Garrido (2004) and Gerber and Shiu (2005) were the first to investigate the GerberShiu function in renewal models. In this paper we will concentrate on a new method for deriving explicit expressions for $m(u)$ in the case of renewal models. In the renewal context, explicit expressions are usually restricted to models where the claim size distribution and in particular the interclaim distribution are (a subclass of) distributions with rational Laplace transform (which includes Erlang and phase-type distributions as well as mixtures of these); see also Willmot (1999); Li and Garrido (2005b). Our method is perfectly suitable for this class of distributions. 
The established methods for deriving explicit expressions for functions arising in risk theory (e.g. ruin probability, Laplace transform of the time to ruin, Gerber-Shiu function) are either based on defective renewal equations or integral equations (Volterra of second kind). Specifically, starting with the defective renewal equation satisfied by the Gerber-Shiu function, Lin and Willmot (2000) propose a solution expressed in terms of the tail of a compound geometric distribution. For particular claim sizes (combinations of exponentials, mixture of Erlangs) they derive explicit analytic solutions for this distribution. In Willmot (2007) this defective renewal equation method is adapted to the analysis of renewal risk models with arbitrary distributions. Another strategy, based on the defective renewal equation, was suggested in the classical compound Poisson model by Drekic et al. (2004). They use Mathematica to obtain the moments of the time to ruin, based on the system of defective renewal difference equations derived by Lin and Willmot (2000). In this paper, we introduce an algebraic operator approach with symbolic techniques for deriving explicit expressions for Gerber-Shiu functions. These techniques are easy to implement, and their further analysis can draw on the full potential of current computer algebra systems.

In general renewal models, $m(u)$ can alternatively be expressed as the solution of a Volterra integral equation of the second kind and hence as a Neumann series, see Gerber and Shiu (1998). Under the further assumption that the interclaim times have rational Laplace transform, the integral equation can be transformed into an integro-differential equation (IDE) with suitable boundary conditions. For the solution of the IDE, due to its convolution structure, Laplace transforms are often the key tool to derive explicit solutions; see e.g. Cheng and Tang (2003), Albrecher and Boxma (2005) and Li and Garrido (2005b). Landriault and Willmot (2008) obtain explicit expressions for the Laplace transform that can be inverted back by partial fractions, for arbitrary interclaim times and Coxian claim sizes. However, explicitly inverting the Laplace transform is in general difficult. Li and Garrido (2004) solved the IDE for $\operatorname{Erlang}(n)[\mathrm{E}(n)]$ (sum of $n$ independent exponential random variables) interclaim times by repeatedly integrating the integro-differential equation satisfied by the Gerber-Shiu function.

In the present paper, we want to advocate an alternative approach to derive explicit expressions for the Gerber-Shiu function in renewal models. For interclaim time distributions with rational Laplace transform - or equivalently if the interclaim density satisfies a linear ordinary differential equations (LODE) with constant coefficients - we first use the systematic approach of Constantinescu (2006) to transform the integral equation for $m(u)$ into an integro-differential equation. If the claim size distribution also has a rational Laplace transform, the IDE can be further reduced to a linear boundary 
value problem with appropriate boundary conditions (Section 2). Evaluating the IDE and its derivatives at 0 and imposing regularity conditions at $\infty$, we supplement the differential equation with sufficiently many boundary conditions so that the Gerber-Shiu function is uniquely determined. This program considerably extends the approach of Chen et al. (2007), who derived a LODE for $m(u)$ in a Poisson jump-diffusion process with phase-type jumps and solved it explicitly for penalty functions that depend only on the deficit at ruin.

Having arrived at a linear boundary problem, we employ the symbolic method developed in Rosenkranz (2005) and Rosenkranz and Regensburger (2008) for computing the integral operator (Green's operator) that maps the penalty function to the corresponding Gerber-Shiu function; see Section 3 for a brief description of this approach. Based on an algebraic operator framework, this method uses noncommutative Gröbner bases for transforming integro-differential and boundary operators to normal forms.

Whereas the classical version of this method works only for boundary value problems on compact intervals, we extend the approach to problems on the positive half-line in Section 4. There we consider operators on functions vanishing at infinity, which is the appropriate setup for our purposes.

In Section 5 we present the solution of the boundary value problem in terms of the Green's operator. The method relies on the factorization of the differential operator using the roots of the Lundberg fundamental equation. This factorization is then lifted to the level of boundary value problems: One can iteratively solve a sequence of first-order boundary value problems with appropriate boundary conditions. It turns out that there is a crucial difference between the roots with positive and negative real part and that there are natural links to the so-called Dickson-Hipp operator. Altogether, this approach allows to compute the Gerber-Shiu function up to quadratures.

In previous papers e.g. Li and Garrido (2004) and Chen et al. (2007), the boundary conditions of the IDE are computed recursively in terms of derivatives of $m(u)$ at zero. In Section 6, we use an integrating factor method with different integration bounds and exploit the Vandermonde-type structure of the resulting matrix for directly deriving an explicit expression for each of these boundary values. This in turn makes it possible to arrive at a fully explicit formula for $m(u)$ in terms of the penalty function. An illustration of our method for $\mathrm{E}(n)$ interclaim times with $\mathrm{E}(m)$ claim sizes is given in Section [7. The method also covers more general models like the case of renewal risk models perturbed by a Brownian motion treated in Section 8 . We conclude in Section 9 by discussing possible extensions of this approach. 


\section{Reduction to a Boundary Value Problem}

Consider $T_{1}$ to be the epoch of the first claim. Since ruin cannot occur in the interval $\left(0, T_{1}\right)$, by the standard renewal argument of Feller (1971, p.183-184) one has

$$
\begin{aligned}
m(u) & =\mathbb{E}\left(e^{-\delta T_{1}} m\left(u+c T_{1}-X_{1}\right)\right) \\
& =\int_{0}^{\infty} e^{-\delta t} f_{\tau}(t)\left(\int_{0}^{u+c t} m(u+c t-y)\right. \\
& \left.+\int_{u+c t}^{\infty} w(u+c t, y-u-c t)\right) f_{X}(y) d y d t,
\end{aligned}
$$

for any claim size density $f_{X}$ and interclaim time density $f_{\tau}$. Due to the net profit condition, the model satisfies the regularity condition

$$
\lim _{u \rightarrow \infty} m(u)=0 .
$$

Define the polynomial

$$
p_{\tau}(x)=x^{n}+a_{n-1} x^{n-1}+\ldots+a_{0},
$$

where $a_{j}$ are real numbers for $j=0,1, \ldots, n$, and $a_{0} \neq 0$. Assume that $f_{\tau}$ satisfies a linear ordinary differential equation with constant coefficients, compactly written in operator notation as

$$
p_{\tau}\left(\frac{d}{d t}\right) f_{\tau}(t)=0
$$

where $\frac{d}{d t}$ is the differentiation operator. For convenience, we consider those LODE representations of $f_{\tau}$ with almost homogeneous initial conditions

$$
\begin{aligned}
f_{\tau}^{(k)}(0) & =0 \quad(k=0, \ldots, n-2), \\
f_{\tau}^{(n-1)}(0) & =a_{0} .
\end{aligned}
$$

The Laplace transform of such a distribution is a rational function that has only a constant as the numerator.

Remark 1. One can express any density which is a convolution of $n$ exponential densities with parameters $\lambda_{i}$ in the above way, namely the polynomial (3) is

$$
p_{\tau}(x)=\prod_{i=1}^{n}\left(x+\lambda_{i}\right)
$$


with almost homogenous initial conditions (5). In the special case of exponentials with the same parameter $\lambda$, this is an Erlang $(n)$ density $f_{\tau}(t)=$ $\frac{1}{(n-1) !} \lambda^{n} t^{n-1} e^{-\lambda t}$, satisfying equation (4) with almost homogenous initial conditions (5) and polynomial

$$
p_{\tau}(x)=(x+\lambda)^{n} .
$$

Under assumption (41) one can now use the technique of integration by parts as in Theorem 3 of Constantinescu (2006, Sec. 3.2) to obtain from (11) the integro-differential equation

$$
p_{\tau}^{*}\left(c \frac{d}{d u}-\delta\right) m(u)=a_{0} \int_{0}^{u} m(u-y) d F_{X}(y)+a_{0} \omega(u),
$$

where the derivatives of $m$ are assumed to exist and to be bounded. Here $\omega(u)=\int_{u}^{\infty} w(u, y-u) d F_{X}(y)$ and

$$
p_{\tau}^{*}(x)=(-1)^{n} x^{n}+(-1)^{n-1} a_{n-1} x^{n-1}+\ldots+a_{0},
$$

where $p_{\tau}^{*}\left(\frac{d}{d t}\right)$ denotes the adjoint operator of the operator $p_{\tau}\left(\frac{d}{d t}\right)$ defined through $\left\langle p_{\tau}\left(\frac{d}{d t}\right) f, g\right\rangle=\left\langle f, p_{\tau}^{*}\left(\frac{d}{d t}\right) g\right\rangle$ with $\langle f, g\rangle=\int_{0}^{\infty} f(x) g(x) d x$ together with (5). In addition to the model regularity condition (2), we will derive in Section [6] the initial values $M_{i}(i=0, \ldots, n-1)$ of the IDE (8) through a variation of the classical integrating factor method of Gerber and Shiu (1998), obtaining

$$
m(0)=M_{0}, m^{\prime}(0)=M_{1}, \ldots, m^{(n-1)}(0)=M_{n-1} .
$$

Together with (2), these boundary conditions make the boundary value problem regular.

Remark 2. Note that the same analysis also works for the case in which the boundary conditions are not of homogeneous type (as for instance would be the case for a mixtures of Erlangs). In that case the Laplace transform of $f_{\tau}$ has a polynomial numerator of lower degree than of the polynomial in the denominator. As a consequence, one obtains further integral terms on the right-hand side of (8), leading to a slightly more cumbersome procedure.

Define the polynomial

$$
p_{X}(x)=x^{n}+b_{n-1} x^{n-1}+\ldots+b_{0} .
$$

If moreover the claim size density $f_{X}$ satisfies a LODE with constant coefficients

$$
p_{X}\left(\frac{d}{d y}\right) f_{X}(y)=0,
$$


and (for simplicity) almost homogeneous boundary conditions

$$
\begin{aligned}
f_{X}^{(k)}(0) & =0 \quad(k=0, \ldots, n-2), \\
f_{X}^{(n-1)}(0) & =b_{0},
\end{aligned}
$$

then the Gerber-Shiu function satisfies a well-posed boundary value problem, namely the LODE

$$
p_{X}\left(\frac{d}{d u}\right) p_{\tau}^{*}\left(c \frac{d}{d u}-\delta\right) m(u)=a_{0} b_{0} m(u)+a_{0} p_{X}\left(\frac{d}{d u}\right) \omega(u)
$$

together with boundary conditions (2) and (9). The characteristic equation

$$
p_{X}(s) p_{\tau}^{*}(c s-\delta)-a_{0} b_{0}=0
$$

of (11) is the Lundberg fundamental equation of this model. Since both the claim sizes and the inter-arrival times have rational Laplace transforms, we know by the results in Li and Garrido (2005a) and Landriault and Willmot (2008) that this equation has exactly $n$ roots with positive and $m$ roots with negative real part as long as $\delta>0$. Note that we exclude the limiting case $\delta=0$, which is equivalent to having 0 as a solution of the Lundberg equation; see Section 5 for a brief discussion of this case.

\section{An Algebraic Operator Approach for Boundary Value Problems}

In order to solve the boundary value problem for (11) we will employ the symbolic computation approach developed in Rosenkranz and Regensburger (2008) and Rosenkranz (2005). As this approach is targeted at boundary value problems for LODE in general differential algebras, we have to extract and adapt the parts needed for our present purposes.

As we can restrict ourselves to LODE with constant coefficients, we first consider two-point boundary value problems on a compact interval $[a, b]$ : Given a forcing function $f(x) \in C[a, b]$, find a solution $g(x) \in C^{n}[a, b]$ of

$$
\begin{aligned}
& \left(D^{n}+c_{n-1} D^{n-1}+\ldots+c_{1} D+c_{0}\right) g=f, \\
& \beta_{1}(g)=\ldots=\beta_{n}(g)=0,
\end{aligned}
$$

where $D=\frac{d}{d x}, c_{i}$ are real numbers and the boundary conditions $\beta_{i}$ are linear combinations of $g(a), \ldots, g^{(n-1)}(a)$ and $g(b), \ldots, g^{(n-1)}(b)$.

Note that the boundary conditions in (13) are homogeneous. As one easily sees, the solution for the general case of inhomogeneous boundary conditions is given by the solution of (13) plus the particular solution of the 
simple boundary value problem with inhomogeneous boundary conditions but $f=0$.

The boundary value problem (13) is called regular if for every $f$ there exists a unique $g$ or equivalently if the associated homogeneous problem only has the trivial solution. This can be checked by testing whether the matrix formed by evaluating the boundary conditions on a fundamental system is regular; for details see Kamke (1967, p. 184). In this case, there is a welldefined operator $G: C[a, b] \rightarrow C^{n}[a, b]$ mapping $f \mapsto g$, known as the Green's operator of (13). While $G$ is usually represented by its associated Green's function (Stakgold, 2000), the operator formulation is more practical in the present setting.

An essential feature of the symbolic operator calculus is that it allows to compose two boundary value problems (in particular those of the form (13)) such that the composite Green's operator is given by the composition of the constituent Green's operators. For solving boundary value problems, the other direction is more important: Any factorization of the underlying differential operator can be lifted to a factorization of boundary value problems. Since we are dealing with differential operators with constant coefficients, we can actually achieve a factorization into first-order boundary value problems. For more details on composing and factoring boundary value problems for LODE, we refer again to Rosenkranz and Regensburger (2008). The theory is developed in an abstract algebraic setting, including in principle also boundary value problems for linear partial differential equations, in Regensburger and Rosenkranz (2009).

In the present setting, we can describe the first-order Green's operators as follows. Writing

$$
A=\int_{a}^{x}, \quad B=\int_{x}^{b}, \quad \text { and } \quad F=\int_{a}^{b}=A+B,
$$

and

$$
A_{\sigma}=e^{\sigma x} A e^{-\sigma x}, \quad B_{\rho}=e^{\rho x} B e^{-\rho x}, \quad \text { and } \quad F_{\sigma \rho}=e^{\sigma x} F e^{-\rho x}
$$

for $\rho, \sigma \in \mathbb{C}$, the basic first-order boundary value problems, with respect to each of the end points of the interval, $(D-\sigma) g=f, g(a)=0$ and $(D-\rho) g=-f, g(b)=0$, have respectively $A_{\sigma}$ and $B_{\rho}$ as their Green's operators as one can see by the fundamental theorem of calculus. Written as operator identities, this means in particular that

$$
\left\{\begin{array}{c}
(D-\sigma) A_{\sigma}=1, \\
(D-\rho) B_{\rho}=-1,
\end{array}\right.
$$

so $A_{\sigma}$ and $-B_{\rho}$ are right inverses of respectively $D-\sigma$ and $D-\rho$ on $C[a, b]$. 
By Rosenkranz (2005, Table 1), we obtain furthermore for any $\tilde{\rho}, \tilde{\sigma} \in \mathbb{C}$

$$
\left\{\begin{aligned}
(\sigma-\tilde{\sigma}) A_{\sigma} A_{\tilde{\sigma}} & =A_{\sigma}-A_{\tilde{\sigma}} \\
(\tilde{\rho}-\rho) B_{\rho} B_{\tilde{\rho}} & =B_{\rho}-B_{\tilde{\rho}} \\
(\rho-\sigma) A_{\sigma} B_{\rho} & =A_{\sigma}+B_{\rho}-F_{\sigma \rho}
\end{aligned}\right.
$$

on $C[a, b]$; the first two are called resolvent identities (Yosida, 1995). For the extension to non-compact intervals in Section 4 we mention an alternative, purely algebraic, way to derive (15), namely as a consequence of conditions that will be simpler to establish in the more general case:

Lemma 1. The identities (15) are algebraic consequences of

$$
\left\{\begin{array}{l}
A_{\sigma}(D-\sigma) A_{\tilde{\sigma}}=A_{\tilde{\sigma}} \\
B_{\rho}(D-\rho) B_{\tilde{\rho}}=-B_{\tilde{\rho}} \\
A_{\sigma}(D-\sigma) B_{\rho}=B_{\rho}-F_{\sigma \rho}
\end{array}\right.
$$

and the identities (14).

Proof. By (14), we have $A_{\sigma}=A_{\sigma}(D-\tilde{\sigma}) A_{\tilde{\sigma}}=A_{\sigma}(\sigma-\tilde{\sigma}+D-\sigma) A_{\tilde{\sigma}}$, which equals $(\sigma-\tilde{\sigma}) A_{\sigma} A_{\tilde{\sigma}}+A_{\tilde{\sigma}}$ because of (16); analogously for the other two identities of (15).

\section{Operators on Functions Vanishing at Infinity}

In the next section, we need the case $a=0$ and $b=\infty$. So we consider the Banach algebra $\left(\mathcal{C}_{0},\|\cdot\|_{\infty}\right)$ of all continuous functions $f:[0, \infty) \rightarrow \mathbb{C}$ vanishing at infinity (Conwav, 1990, p. 65). The subalgebra of $\mathcal{C}_{0}$ consisting of $n$-times continuously differentiable functions is denoted by $\mathcal{C}_{0}^{n}$. The following proposition makes precise in how far the situation on $C[a, b]$ carries over to $\mathcal{C}_{0}$; confer also Butzer and Berens (1967, Prop. 1.3.12) for the case of bounded uniformly continuous functions on $\mathbb{R}$.

Proposition 2. For $\rho \in \mathbb{C}$ with $\operatorname{Re}(\rho)>0$, we have continuous integral operators

$$
A_{-\rho}, B_{\rho}, e^{-\rho x} A, B e^{-\rho x}: \quad \mathcal{C}_{0} \rightarrow \mathcal{C}_{0}^{1}
$$

with norm bounded by $1 / \operatorname{Re}(\rho)$, and the identities (14), (15) are valid for all $\rho, \tilde{\rho}, \sigma, \tilde{\sigma} \in \mathbb{C}$ with $\operatorname{Re}(\rho), \operatorname{Re}(\tilde{\rho})>0$ and $\operatorname{Re}(\sigma), \operatorname{Re}(\tilde{\sigma})<0$.

Proof. Let $\eta=\operatorname{Re}(\rho)$. We first check that the operators (17) map $\mathcal{C}_{0}$ into $\mathcal{C}_{0}$. For $A_{-\rho}$ we use that

$$
\left|A_{-\rho} f(x)\right| \leq e^{-\eta x} \int_{0}^{y} e^{\eta \xi}|f(\xi)| d \xi+e^{-\eta x} \int_{y}^{x} e^{\eta \xi}|f(\xi)| d \xi
$$


for all $f \in \mathcal{C}_{0}$ and $x \geq y \geq 0$. Fixing $\varepsilon>0$, the first summand is smaller than $\varepsilon / 2$ for $x \geq x_{0}(\varepsilon, y)$ because $\eta>0$. Since $f \in \mathcal{C}_{0}$, we have $|f(\xi)|<\varepsilon \eta / 2$ for all $\xi \geq y_{0}(\varepsilon)$, so the second summand is smaller than $\varepsilon / 2$ for $x \geq y_{0}(\varepsilon)$ and $y=y_{0}(\varepsilon)$. Thus we obtain $\left|A_{-\rho} f(x)\right|<\varepsilon$ for all $x \geq \max \left\{y_{0}(\varepsilon), x_{0}\left(\varepsilon, y_{0}(\varepsilon)\right)\right\}$. Using a similar argument as for the second summand, we obtain $B_{\rho} f \in \mathcal{C}_{0}$. One immediately checks that $e^{-\rho x} A$ and $B e^{-\rho x}$ map even bounded functions into $\mathcal{C}_{0}$.

Next we verify that the operators are continuous. The norm bound for $A_{-\rho}$ follows from $\left|A_{-\rho} f(x)\right| \leq e^{-\eta x}\|f\|_{\infty} \int_{0}^{x} e^{\eta \xi} d \xi$ and $e^{-\eta x} \int_{0}^{x} e^{\eta \xi} d \xi \leq 1 / \eta$; similarly for $e^{-\rho x} A$ and $B e^{-\rho x}$. For $B_{\rho}$ we use the representation

$$
B_{\rho} f(x)=\int_{0}^{\infty} e^{-\rho \xi} f(\xi+x) d \xi
$$

and the fact that $\int_{0}^{\infty} e^{-\eta \xi} d \xi=1 / \eta$.

Now we turn to differentiability and identities (14). For $A_{-\rho}$ this follows immediately from the fundamental theorem of calculus. Using representation (18), the difference quotient $\left(B_{\rho} f(x+h)-B_{\rho} f(x)\right) / h$ is given by

$$
\frac{e^{\rho h}-1}{h} \int_{h}^{\infty} e^{-\rho \xi} f(\xi+x) d \xi-\frac{1}{h} \int_{0}^{h} e^{-\rho \xi} f(\xi+x) d \xi
$$

which converges to $\rho B_{\rho} f(x)-f(x)$ as $h \rightarrow 0$. Finally, $e^{-\rho x} A f$ is differentiable again by the fundamental theorem and $B e^{-\rho x} f=e^{-\rho x} B_{\rho} f$ is differentiable because $B_{\rho} f$ is by what we have just seen.

It remains to prove the identities (15), (16); by Lemma 1 it suffices to show the latter. These are an easy consequence of the fact that

$$
A_{\sigma}(D-\sigma) f(x)=f(x)-e^{\sigma x} f(0) \quad \text { and } \quad B_{\rho}(D-\rho) f(x)=-f(x)
$$

for all $f \in \mathcal{C}_{0}^{1}$. The identity for $A_{\sigma}$ carries over from the bounded case and is even valid on $C^{1}[0, \infty)$, the one for $B_{\rho}$ follows from the representation (18) and integration by parts.

Remark 3. Note that $B_{o}$ also appears in the literature as the Dickson-Hipp operator (Dickson and Hipp, 2001; Li and Garrido, 2004), and the second equation of (15) is also used in these papers. The crucial contribution of the present result is the third equation of (15), i.e. the interaction between the Dickson-Hipp operator $B_{\rho}$ and its counterpart $A_{\sigma}$.

We write $\mathcal{E}_{0} \subset \mathcal{C}_{0}$ for the subalgebra of exponential polynomials spanned by $x^{j} e^{-\rho x}$ with $\operatorname{Re}(\rho)>0$. 
Proposition 3. The subalgebra $\mathcal{E}_{0}$ is dense in $\mathcal{C}_{0}$, and the operators (17) map $\mathcal{E}_{0}$ into itself.

Proof. Density follows from the Stone-Weierstrass Theorem for locally compact spaces (Conwav, 1990, p. 147). For proving that the operators (17) map $\mathcal{E}_{0}$ into itself, one uses induction on $j$ and integration by parts.

Note that - by the same reasoning - the operators $A_{\rho}$ and $B_{\rho}$ also map $\mathcal{E}_{0}$ into itself if $\operatorname{Re}(\rho)=0$ but they are no longer continuous.

This proposition provides an alternative approach to proving the identities (14), (15): Since $\mathcal{E}_{0}$ is dense in $\mathcal{C}_{0}$ and the operators are continuous, it suffices to prove them for exponential polynomials - this can be done by an elementary computation and induction on $j$. Density arguments of this type could also be useful for generalizing to larger function spaces like $L^{p}$ or spaces based on regular variation (Bingham et al., 1987).

\section{Solving Boundary Value Problems on the Half-Line}

For computing the Gerber-Shiu function, the method described in Section 2 leads to a boundary value problem on the half-line. In fact, we can rewrite equation (11) as

$$
T m=f
$$

with

$$
T=p_{X}\left(\frac{d}{d u}\right) p_{\tau}^{*}\left(c \frac{d}{d u}-\delta\right)-a_{0} b_{0} \quad \text { and } \quad f(u)=a_{0} p_{X}\left(\frac{d}{d u}\right) \omega(u),
$$

initial values $m^{(i)}(0)=M_{i}$, and regularity condition $m(\infty)=0$. As noted earlier (beginning of Section 3), it suffices to consider the corresponding homogeneous boundary conditions and incorporate the boundary values in specific settings afterwards (Sections 7 and 8).

So let us now consider the general boundary value problem on the half-line with homogeneous boundary conditions,

$$
\begin{aligned}
& T g=f \\
& g(0)=\ldots=g^{(m-1)}(0)=0 \text { and } \quad g \in \mathcal{C}_{0},
\end{aligned}
$$

where the forcing function $f$ is required to vanish at infinity.

We assume that the characteristic equation of $T$ has distinct roots, which we divide into $\rho_{1}, \ldots, \rho_{n}$ with positive and $\sigma_{1}, \ldots, \sigma_{m}$ with negative real part (for the case of roots with zero real part see the discussion at the end of the section). Thus we have the differential operator $T=T_{\rho} T_{\sigma}$ with

$$
T_{\rho}=\left(D-\rho_{1}\right) \cdots\left(D-\rho_{n}\right) \quad \text { and } \quad T_{\sigma}=\left(D-\sigma_{1}\right) \cdots\left(D-\sigma_{m}\right) .
$$


Note that in order to have a regular boundary value problem, it is sufficient to prescribe $m$ initial conditions even though the order of $T$ is $m+n$. This is due to the regularity condition $g \in \mathcal{C}_{0}$ : The general solution $g$ of the associated homogeneous differential equation $T g=0$ is a linear combination of $e^{\rho_{j} x}$ and $e^{\sigma_{i} x}$, where all terms with positive roots must vanish and the remaining $m$ coefficients are determined by the $m$ conditions at zero.

The crucial point is that it is possible to factor this boundary value problem along $T=T_{\rho} T_{\sigma}$ into the regular boundary value problems

$$
\begin{aligned}
& T_{\sigma} g=h, \quad \text { and } \quad T_{\rho} h=f \text {, } \\
& g(0)=\ldots=g^{(m-1)}(0)=0 \quad \text { and } \quad h \in \mathcal{C}_{0}
\end{aligned}
$$

with forcing function $f \in \mathcal{C}_{0}$.

Lemma 4. The boundary value problems (21) have

$$
G_{\sigma}=A_{\sigma_{1}} \cdots A_{\sigma_{m}}=\sum_{i=1}^{m} a_{i} A_{\sigma_{i}} \quad \text { and } \quad G_{\rho}=(-1)^{n} B_{\rho_{1}} \cdots B_{\rho_{n}}=\sum_{j=1}^{n} b_{j} B_{\rho_{j}}
$$

with

$$
a_{i}=\prod_{k=1, k \neq i}^{m}\left(\sigma_{i}-\sigma_{k}\right)^{-1} \quad \text { and } \quad b_{j}=-\prod_{k=1, k \neq j}^{n}\left(\rho_{j}-\rho_{k}\right)^{-1}
$$

as their Green's operators, so $g=G_{\sigma} h$ and $h=G_{\rho} f$, where $\prod_{k=1, k \neq 1}^{1}=1$.

Proof. Let us first prove the identity for $G_{\sigma}$ by induction (the case for $G_{\rho}$ is analogous). The base case $m=1$ is trivial, so assume the identity for $m-1$. Then (15) yields

$$
A_{\sigma_{1}} \cdots A_{\sigma_{m-1}} A_{\sigma_{m}}=\sum_{i=1}^{m-1} a_{i} A_{\sigma_{i}}-\left(\sum_{i=1}^{m-1} \prod_{k=1, k \neq i}^{m}\left(\sigma_{i}-\sigma_{k}\right)^{-1}\right) A_{\sigma_{m}}
$$

and we are done since the parenthesis is equal to $-a_{m}$ by the well-known partial fraction formula.

By Proposition 2, the Green's operators $G_{\rho}$ and $G_{\sigma}$ map $\mathcal{C}_{0}$ to $\mathcal{C}_{0}^{m}$ and $\mathcal{C}_{0}^{n}$, respectively, and (14) yields $T_{\sigma} G_{\sigma}=1$ and $T_{\rho} G_{\rho}=1$. It remains to check that $G_{\sigma} f$ satisfies the initial conditions. For that we prove for all $i<m$ the identity

$$
D^{i} G_{\sigma}=\sum_{l=0}^{i} h_{i-l}\left(\sigma_{1}, \ldots, \sigma_{l+1}\right) A_{\sigma_{l+1}} \cdots A_{\sigma_{m}},
$$

where $h_{i-l}$ denotes the complete homogeneous symmetric polynomial of degree $i-l$ in the indicated variables (Stanlev, 1999, p. 294); the claim then 
follows because $A_{\sigma_{1}} f(0), \ldots, A_{\sigma_{m}} f(0)=0$. The base case $i=0$ is trivial, so assume (22) for $i-1$. Using $D A_{\sigma_{l+1}}=1+\sigma_{l+1} A_{\sigma_{l+1}}$ from (14), this gives

$$
\begin{aligned}
D^{i} G_{\sigma}= & \sum_{l=0}^{i-1} h_{i-l-1}\left(\sigma_{1}, \ldots, \sigma_{l+1}\right) D A_{\sigma_{l+1}} \cdots A_{\sigma_{m}} \\
= & \sum_{l=1}^{i-1}\left(h_{i-l}\left(\sigma_{1}, \ldots, \sigma_{l}\right)+\sigma_{l+1} h_{i-l-1}\left(\sigma_{1}, \ldots, \sigma_{l+1}\right)\right) A_{\sigma_{l+1}} \cdots A_{\sigma_{m}} \\
& \quad+\sigma_{1}^{i} A_{\sigma_{1}} \cdots A_{\sigma_{m}}+A_{\sigma_{i+1}} \cdots A_{\sigma_{m}}
\end{aligned}
$$

after a little rearrangement. But the parenthesized factor in the sum simplifies to $h_{i-l}\left(\sigma_{1}, \ldots, \sigma_{l+1}\right)$, while the outlying summands also have the right factors $h_{i-0}\left(\sigma_{1}\right)=\sigma_{1}^{i}$ and $h_{i-i}\left(\sigma_{1}, \ldots, \sigma_{j+1}\right)=1$, respectively.

Theorem 5. The boundary value problem (20) has the Green's operator

$$
\begin{aligned}
G_{\sigma} G_{\rho} & =\sum_{i=1}^{m} \sum_{j=1}^{n} c_{i j}\left(A_{\sigma_{i}}+B_{\rho_{j}}-F_{\sigma_{i} \rho_{j}}\right) \\
& =\sum_{i=1}^{m} \sum_{j=1}^{n} c_{i j}\left(e^{\sigma_{i} x} A\left(e^{-\sigma_{i} x}-e^{-\rho_{j} x}\right)+\left(e^{\rho_{j} x}-e^{\sigma_{i} x}\right) B e^{-\rho_{j} x}\right)
\end{aligned}
$$

where $c_{i j}=a_{i} b_{j}\left(\rho_{j}-\sigma_{i}\right)^{-1}$, i.e. $g=G_{\sigma} G_{\rho} f$.

Proof. Let $f \in \mathcal{C}_{0}$. From Proposition 2 we know that $G=G_{\sigma} G_{\rho}$ maps $f$ into $\mathcal{C}_{0}^{m+n}$. By the previous lemma, $G f$ satisfies the differential equation and the initial conditions. For proving that $G$ has the indicated sum representations, we use again Lemma 4, the identities (15) and the definition of $F_{\sigma_{i} \rho_{j}}$.

If some of the $\rho_{j}$ have zero real part, the above Green's operator $G$ no longer maps $\mathcal{C}_{0}$ into itself, so the boundary value problems (20) cannot be expected to have a solution for all $f \in \mathcal{C}_{0}$. But if $G f \in \mathcal{C}_{0}$, it is the unique solution of (20); by the observations after Proposition 3, this in particular true for $f \in \mathcal{E}_{0}$.

\section{Initial Values for $\mathrm{E}(\boldsymbol{n})$ Risk Processes}

The next step for solving the boundary value problem for (19) is to determine the initial values $M_{i}$ of (9). We consider the case of $\mathrm{E}(n)$ distributed interclaim times (under assumption that $m$ has bounded derivatives). Using (77) in the integro-differential equation (8), we obtain

$$
\left(-c \frac{d}{d u}+(\lambda+\delta)\right)^{n} m(u)=\lambda^{n} \int_{0}^{u} m(u-y) d F_{X}(y)+\lambda^{n} \omega(u)
$$


with the corresponding Lundberg fundamental equation

$$
(-c z+(\lambda+\delta))^{n}-\lambda^{n} \hat{f}_{X}(z)=0,
$$

where $\hat{f}_{X}(z)=\mathbb{E}\left(e^{-z X}\right)$ is the Laplace transform of $f_{X}(u)$. Equation (24) has exactly $n$ solutions $\rho_{i}(i=1, \ldots, n)$ with positive real part, according to $\mathrm{Li}$ and Garrido (2004).

We will use a similar integrating factors technique as the one proposed in Gerber and Shiu (1998) and arrive at a system of linear equations in the initial values that we can solve explicitly. A different choice of the integration bounds will simplify some steps compared to a related approach of $\mathrm{Li}$ and Garrido (2004). The change of variables and order of integration used in Gerber and Shiu (1998) is then not necessary here. Let us multiply equation (23) by $e^{-\rho_{i} u}$ for each $i=1, \ldots, n$, and then integrate from $u=\infty$ to $u=x$ to arrive at

$$
\begin{aligned}
& \sum_{j=0}^{n}\left(\begin{array}{c}
n \\
j
\end{array}\right)(-c)^{j}(\lambda+\delta)^{(n-j)} \int_{\infty}^{x} e^{-\rho_{i} u} m^{(j)}(u) d u \\
& \quad=\lambda^{n} \int_{\infty}^{x} e^{-\rho_{i} u} \int_{0}^{u} m(u-y) d F_{X}(y) d u+\lambda^{n} \int_{\infty}^{x} e^{-\rho_{i} u} \omega(u) d u .
\end{aligned}
$$

Now we use integration by parts together with

$$
\lim _{u \rightarrow \infty} e^{-\rho_{i} u} m^{(j)}(u)=0 \quad(j=0, \ldots, n, i=1, \ldots, n)
$$

to obtain

$$
\begin{gathered}
\int_{\infty}^{x} e^{-\rho_{i} u} m^{(j)}(u) d u=\sum_{k=0}^{j-1} e^{-\rho_{i} x} \rho_{i}^{k} m^{(j-k-1)}(x)+\rho_{i}^{j} I_{i}(x), \\
\text { where } \quad I_{i}(x)=\int_{\infty}^{x} e^{-\rho_{i} u} m(u) d u .
\end{gathered}
$$

Then evaluating each equation at $x=0$, we note that the left-hand side and the right-hand side terms pertaining to $I_{i}(0)$ cancel due to (24) evaluated at $z=\rho_{i}$. Also we see that in the right-hand side the second integral is actually $-\hat{\omega}\left(\rho_{i}\right)$, the Laplace transform of $\omega$ evaluated at $\rho_{i}$. We obtain a system of $n$ equations in $n$ unknown variables $m^{(k)}(0)$

$$
\sum_{j=1}^{n}\left(\begin{array}{l}
n \\
j
\end{array}\right)(-c)^{j}(\lambda+\delta)^{n-j} \sum_{k=0}^{j-1} \rho_{i}^{k} m^{(j-k-1)}(0)=-\lambda^{n} \hat{\omega}\left(\rho_{i}\right)
$$


for $k=0, \ldots, n-1$. Collecting and rearranging the terms, we get

$$
\sum_{k=0}^{n-1} m^{(k)}(0) \underbrace{\sum_{j=0}^{n-k-1}\left(\begin{array}{l}
n \\
j
\end{array}\right)\left(-\frac{\lambda+\delta}{c}\right)^{j} \rho_{i}^{(n-k-1)-j}}_{p_{n-k-1}\left(\rho_{i}\right)}=-\left(-\frac{\lambda}{c}\right)^{n} \hat{\omega}\left(\rho_{i}\right),
$$

for $i=1, \ldots, n$. Note that the polynomials

$$
p_{k}(x)=\sum_{j=0}^{k}\left(\begin{array}{c}
n \\
k-j
\end{array}\right)\left(-\frac{\lambda+\delta}{c}\right)^{k-j} x^{j}
$$

appearing in the coefficients of $m^{(n-k-1)}(0)$ are monic of degree $k$.

We express the system in matrix form $A x=b$ as

$$
\left(\begin{array}{ccc}
p_{0}\left(\rho_{1}\right) & \cdots & p_{n-1}\left(\rho_{1}\right) \\
\vdots & \ddots & \vdots \\
p_{0}\left(\rho_{n}\right) & \cdots & p_{n-1}\left(\rho_{n}\right)
\end{array}\right)\left(\begin{array}{c}
m^{(n-1)}(0) \\
\vdots \\
m^{(0)}(0)
\end{array}\right)=-\left(-\frac{\lambda}{c}\right)^{n}\left(\begin{array}{c}
\hat{\omega}\left(\rho_{1}\right) \\
\vdots \\
\hat{\omega}\left(\rho_{n}\right)
\end{array}\right) .
$$

According to Cramer's rule, the solution of this system of equations is of the form

$$
m^{(k)}(0)=\frac{\operatorname{det}\left(B_{n-1-k}\right)}{\operatorname{det}(A)} \quad(k=0, \ldots, n-1),
$$

where $B_{k}$ is the $n \times n$ matrix obtained from $A$ by replacing the $(k+1)$-th column of $A$ by the right-hand side $b$.

The following result generalizes the formula for $m(0)$ given in Gerber and Shiu (2005, Eqn. 8.1).

Proposition 6. The $k$-th derivative of the expected discounted penalty function evaluated at zero has the form

$$
m^{(k)}(0)=(-1)^{k}\left(\frac{\lambda}{c}\right)^{n} \sum_{i=1}^{n} \frac{\hat{\omega}\left(\rho_{i}\right) S\left(\rho_{i}^{\prime}, k\right)}{\prod_{l=1, \ldots, n ; l \neq i}\left(\rho_{l}-\rho_{i}\right)}
$$

for $k=0, \ldots, n-1$, where $\rho_{i}^{\prime}=\left(\rho_{1}, \ldots, \rho_{i-1}, \rho_{i+1}, \ldots, \rho_{n}\right)$ and

$$
S\left(\rho_{i}^{\prime}, k\right)=\sum_{j=0}^{k}\left(-\frac{\lambda+\delta}{c}\right)^{j}\left(\begin{array}{c}
n-1+j \\
j
\end{array}\right) e_{k-j}\left(\rho_{i}^{\prime}\right),
$$

with $e_{k}$ the elementary symmetric polynomials of degree $k$. 
Proof. According to Krattenthalen (1999), the determinant of the matrix $A$ is the same as the Vandermonde determinant $V_{n}=V_{n}\left(\rho_{1}, \ldots, \rho_{n}\right)$ so

$$
\operatorname{det}(A)=\prod_{1 \leq i<j \leq n}\left(\rho_{j}-\rho_{i}\right)
$$

We will show that the determinant of $B_{k}$ is the product of a Vandermonde determinant and a linear combination of symmetric polynomials in the $\rho_{i}$ and $\hat{\omega}\left(\rho_{i}\right)$. Expanding along the $(n-k)$-th column, one gets

$$
\operatorname{det}\left(B_{n-1-k}\right)=\sum_{i=1}^{n}(-1)^{i+n-k} b_{i} \operatorname{det}\left(A_{i, n-k}\right)
$$

where $A_{i, k}$ is the $(n-1) \times(n-1)$ matrix obtained from $A$ by removing the $i$-th row and the $k$-th column. By applying Corollary A2 of the appendix to the matrix $A_{i, k}$ and observing that

$$
q(x)=\left(1-\frac{\lambda+\delta}{c} x\right)^{n}-\left(1+\left(-\frac{\lambda+\delta}{c} x\right)^{n}\right),
$$

we obtain

$$
\operatorname{det}\left(A_{i, n-k}\right)=V_{n-1}\left(\rho_{i}^{\prime}\right) \sum_{j=0}^{k} d_{j} e_{k-j}\left(\rho_{i}^{\prime}\right)
$$

where

$$
d_{j}=\left[x^{j}\right] \frac{(-1)^{j}+\left(\left(1-\frac{\lambda+\delta}{c} x\right)^{n}-\left(1+\left(-\frac{\lambda+\delta}{c} x\right)^{n}\right)\right)^{j+1}}{\left(1-\frac{\lambda+\delta}{c} x\right)^{n}-\left(-\frac{\lambda+\delta}{c} x\right)^{n}}
$$

and $\left[x^{j}\right] f(x)=f^{(j)}(0) / j$ ! denotes the coefficient of $x^{j}$ of a power series $f(x)$. We will show below that

$$
d_{j}=\left(-\frac{\lambda+\delta}{c}\right)^{j}\left(\begin{array}{c}
n-1+j \\
j
\end{array}\right) .
$$

Inserting the resulting formula for the determinant $A_{i, k}$ into the expansion of $\operatorname{det}\left(B_{k}\right)$ in Cramer's rule, we get

$$
m^{(k)}(0)=\left(-\frac{\lambda}{c}\right)^{n} \sum_{i=1}^{n}(-1)^{i+n-k+1} \hat{\omega}\left(\rho_{i}\right) \frac{V_{n-1}\left(\rho_{i}^{\prime}\right)}{V_{n}} \sum_{j=0}^{k} d_{j} e_{k-j}\left(\rho_{i}^{\prime}\right),
$$

which after cancellation of the Vandermode terms leads to the result stated. 
It remains to show equation (29). From equation (34) we get that $(-1)^{j} d_{j}=$ $\left[x^{j}\right] \sum_{m=0}^{j}(-q(x))^{m}$. Since $j<n$ we can safely add terms of order at least $n$ to $q(x)$. We do this and replace $q(x)$ with $\left(1-\frac{\lambda+\delta}{c} x\right)^{n}-1$. Inserting the modified $q(x)$ and expanding the expression, we obtain

$$
\begin{aligned}
\sum_{m=0}^{j}(-q(x))^{m} & =\sum_{m=0}^{j}(-1)^{m}\left(\left(1-\frac{\lambda+\delta}{c} x\right)^{n}-1\right)^{m} \\
& =\sum_{m=0}^{j} \sum_{l=0}^{m}\left(\begin{array}{c}
m \\
l
\end{array}\right)(-1)^{l} \sum_{h=0}^{n l}\left(\begin{array}{c}
n l \\
h
\end{array}\right)\left(-\frac{\lambda+\delta}{c} x\right)^{h} \\
& =\sum_{h=0}^{n m}\left(-\frac{\lambda+\delta}{c} x\right)^{h} \sum_{m=0}^{j} \sum_{l=0}^{m}(-1)^{l}\left(\begin{array}{c}
m \\
l
\end{array}\right)\left(\begin{array}{c}
n l \\
h
\end{array}\right),
\end{aligned}
$$

so that $d_{j}=\left(\frac{\lambda+\delta}{c}\right)^{j} \sum_{m=0}^{j} \sum_{l=0}^{m}(-1)^{l}\left(\begin{array}{c}m \\ l\end{array}\right)\left(\begin{array}{c}n l \\ j\end{array}\right)$. Rearranging and using the simple binomial identities of Graham et al. (1989, 5.10 and 5.14), we can simplify the double sum to

$$
\begin{aligned}
& \sum_{l=0}^{j}(-1)^{l}\left(\begin{array}{c}
n l \\
j
\end{array}\right) \sum_{m=0}^{j}\left(\begin{array}{c}
m \\
l
\end{array}\right)=\sum_{l=0}^{j}(-1)^{l}\left(\begin{array}{c}
j+1 \\
l+1
\end{array}\right)\left(\begin{array}{c}
n l \\
j
\end{array}\right) \\
= & \sum_{l=0}^{j+1}(-1)^{l+1}\left(\begin{array}{c}
j+1 \\
l
\end{array}\right)\left(\begin{array}{c}
n(l-1) \\
j
\end{array}\right)+\left(\begin{array}{c}
-n \\
j
\end{array}\right) \\
= & (-1)^{j}\left(\begin{array}{c}
n-1+j \\
j
\end{array}\right)-\sum_{l=0}^{j+1}(-1)^{l}\left(\begin{array}{c}
j+1 \\
l
\end{array}\right)\left(\begin{array}{c}
n(l-1) \\
j
\end{array}\right) .
\end{aligned}
$$

Finally, the last sum vanishes due to Graham et al. (1989, 5.42) since it is the $(j+1)$-th difference of $\left(\begin{array}{c}n(l-1) \\ j\end{array}\right)$ as a polynomial in $l$, which is only of degree $j$.

Since the Gerber-Shiu function is the unique solution of (19), it has the form

$$
m(u)=G_{\sigma} G_{\rho} f(u)+m^{p}(u),
$$

where $G_{\sigma} G_{\rho}$ is given in Theorem [5 and $m^{p}(u)$ is the particular solution obtained as a linear combination of the $e^{\sigma_{i} u}$, with factors determined by the initial values from Proposition [6.

\section{Explicit Solution for E $(n)$ Risk Processes with E $(m)$ Claims}

Let us now specialize the differential equation (11) for the Gerber-Shiu function to the case of $\operatorname{Erlang}(n, \lambda)$ interclaim times and $\operatorname{Erlang}(m, \mu)$ claim sizes, 
with discount rate $\delta>0$. From the previous section we get $n$ boundary conditions. As described in Section 5 one in fact needs $m$ boundary conditions, so we assume $m \leq n$ (otherwise, one can derive the remaining conditions by evaluating higher derivatives of the integro-differential equation (23)). We obtain a boundary value problem for the differential equation $T m=f$ with $D=\frac{d}{d u}$, where

$$
\begin{gathered}
T=(D+\mu)^{m}(-c D+\lambda+\delta)^{n}-\lambda^{n} \mu^{m} \\
f(u)=\frac{\lambda^{n} \mu^{m}}{(m-1) !}(D+\mu)^{m} \int_{u}^{\infty} w(u, y-u) y^{m-1} e^{-\mu y} d y
\end{gathered}
$$

and boundary conditions (2) and (9). To apply the results from Section 5 , we can choose any sufficiently smooth penalty function $w(x, y)$ such that $\lim _{u \rightarrow \infty} f(u)=0$. By Proposition 3 this includes all bivariate exponential polynomials whose terms $x^{i} y^{j} e^{\alpha x} e^{\beta y}$ satisfy $\alpha<\beta<\mu$.

Since the characteristic equation for $T$ is the Lundberg fundamental equation, we know from the general results mentioned in Section 2 that it has $n$ roots $\rho_{1}, \ldots, \rho_{n}$ with positive real part and $m$ roots $\sigma_{1}, \ldots, \sigma_{m}$ with negative real part. So we have the factorization

$$
T=T_{\rho} T_{\sigma}=\left(D-\rho_{1}\right) \cdots\left(D-\rho_{n}\right)\left(D-\sigma_{1}\right) \cdots\left(D-\sigma_{m}\right),
$$

and Theorem 5 gives us the Green's operator for the corresponding homogeneous boundary value problem.

Writing $\hat{f}$ for the Laplace transform of $f$ and using the definition of the corresponding operators, we obtain from Theorem 5 the explicit form of the Gerber-Shiu function

$m(u)=\sum_{i=1}^{m} \sum_{j=1}^{n} c_{i j}\left(\left(\int_{0}^{u} e^{\sigma_{i}(u-\xi)}+\int_{u}^{\infty} e^{\rho_{j}(u-\xi)}\right) f(\xi) d \xi-\hat{f}\left(\rho_{j}\right) e^{\sigma_{i} u}\right)+m^{p}(u)$

with

$$
c_{i j}=-\prod_{k=1, k \neq i}^{m}\left(\sigma_{i}-\sigma_{k}\right)^{-1} \prod_{k=1, k \neq j}^{n}\left(\rho_{j}-\rho_{k}\right)^{-1}\left(\rho_{j}-\sigma_{i}\right)^{-1} .
$$

With the initial values from formula (28) the computation of the particular solution $m^{p}$ satisfying the inhomogeneous boundary conditions reduces to solving a system of linear equations, obtained from imposing the condition that the particular solution satisfies these given initial conditions, i.e. $\left(m^{p}\right)^{(i)}(0)=M_{i}$. As remarked in Section 5, formula (31) remains valid for suitable $f$ also in the limiting case $\delta=0$, which is equivalent to having 0 among the $\rho_{1}, \ldots, \rho_{n}$. 
So the problem of computing the Gerber-Shiu function for a given penalty function is reduced to quadratures: Since symbolic algorithms for evaluating one-dimensional integrals are very powerful (Bronstein, 2005) and easily accessible in current computer algebra systems, one will often obtain an explicit expression for the Gerber-Shiu function. Otherwise one can resort to standard numerical methods for obtaining approximations.

In the particular case $n=2, m=1$ one has

$$
\begin{aligned}
T & =(D+\mu)(-c D+\lambda+\delta)^{2}-\lambda^{2} \mu, \\
f(u) & =\lambda^{2} \mu(D+\mu) \int_{u}^{\infty} w(u, y-u) e^{-\mu y} d y .
\end{aligned}
$$

After calculating the particular solution using the initial value from Proposition [6. we obtain the Gerber-Shiu function in the explicit form

$$
\begin{aligned}
m(u) & =\frac{e^{\sigma u}}{\rho_{1}-\rho_{2}}\left(\frac{\hat{f}\left(\rho_{1}\right)}{\rho_{1}-\sigma}-\frac{\hat{f}\left(\rho_{2}\right)}{\rho_{2}-\sigma}-\left(\frac{\lambda}{c}\right)^{2}\left(\hat{\omega}\left(\rho_{1}\right)-\hat{\omega}\left(\rho_{2}\right)\right)\right) \\
& -\frac{1}{\rho_{1}-\rho_{2}} \int_{u}^{\infty}\left(\frac{1}{\rho_{1}-\sigma} e^{\rho_{1}(u-\xi)}-\frac{1}{\rho_{2}-\sigma} e^{\rho_{2}(u-\xi)}\right) f(\xi) d \xi \\
& +\frac{1}{\rho_{1}-\sigma} \frac{1}{\rho_{2}-\sigma} \int_{0}^{u} e^{\sigma(u-\xi)} f(\xi) d \xi,
\end{aligned}
$$

where one should recall that $\rho_{1}, \rho_{2}$ are the positive roots and $\sigma$ is the negative root of the fundamental Lundberg equation. For example, when $w(x, y)=$ $x^{j} y^{k}$ with $j$ and $k$ positive integers, one obtains

$$
\begin{aligned}
& \frac{\Delta \mu^{k}}{k ! \lambda^{2}} m(u)=-\frac{\rho_{2}-\sigma}{\left(\rho_{1}+\mu\right)^{j}}\left(j \Gamma\left(j,\left(\rho_{1}+\mu\right) u\right) e^{\rho_{1} u}+\frac{j !}{c^{2}}\left(\frac{\rho_{1}-\sigma}{\rho_{1}+\mu}-c^{2}\right) e^{\sigma u}\right) \\
& \quad+\frac{\rho_{1}-\sigma}{\left(\rho_{2}+\mu\right)^{j}}\left(j \Gamma\left(j,\left(\rho_{2}+\mu\right) u\right) e^{\rho_{2} u}+\frac{j !}{c^{2}}\left(\frac{\rho_{2}-\sigma}{\rho_{2}+\mu}-c^{2}\right) e^{\sigma u}\right) \\
& \quad-\frac{\rho_{1}-\rho_{2}}{(\sigma+\mu)^{j}}(j \Gamma(j,(\sigma+\mu) u)-j !) e^{\sigma u}
\end{aligned}
$$

where $\Delta=\left(\rho_{1}-\rho_{2}\right)\left(\rho_{1}-\sigma\right)\left(\rho_{2}-\sigma\right)$ is the square root of the discriminant associated to the fundamental Lunderberg equation and $\Gamma(a, x)=$ $\int_{x}^{\infty} t^{a-1} e^{-t} d t$ is the incomplete Gamma function. This formula extends Equation (3.8) of Cheng and Tang (2003) and similar examples with $n=2$ from Li and Garrido (2004, 2005b) and Gerber and Shiu (2005).

\section{Explicit Solution for the Classical Perturbed Risk Model}

For the case of an $\operatorname{Erlang}(n, \lambda)$ risk model perturbed by a Brownian motion, the Gerber-Shiu function satisfies an integro-differential equation as given in Constantinescu (2006) 


$$
\left(-\frac{\tilde{\sigma}^{2}}{2} \frac{d^{2}}{d u^{2}}-c \frac{d}{d u}+\lambda+\delta\right)^{n} m(u)=\lambda^{n} \int_{0}^{u} m(u-x) f_{X}(x) d x+\lambda^{n} \omega(u),
$$

where $\tilde{\sigma}$ is the diffusion coefficient. Since the differential operator of this equation has constant coefficients, the method introduced in this paper applies. As before, for claim distributions with rational Laplace transform, the equation reduces to a LODE. For instance, in the case of $\mathrm{E}(m, \mu)$ claim sizes, this LODE has the same form $T m=f$ with $D=\frac{d}{d u}$, with

$$
T=(D+\mu)^{m}\left(-\frac{\tilde{\sigma}^{2}}{2} D^{2}-c D+\lambda+\delta\right)^{n}-\lambda^{n} \mu^{m},
$$

and $f(u)$ as in (30) and the appropriate boundary conditions. The characteristic equation for $T$ is again the fundamental Lundberg equation.

Also in this case we can derive explicit expressions for the Gerber-Shiu function. To exemplify, we consider the well-known case of a compound Poisson process perturbed by a Brownian motion with exponential claim sizes, $\mathrm{E}(1, \lambda)-\mathrm{E}(1, \mu)$ in the notation introduced here. Then the LODE is of order three, with

$$
T=(D+\mu)\left(-\frac{\tilde{\sigma}^{2}}{2} D^{2}-c D+\lambda+\delta\right)-\lambda \mu
$$

and

$$
f(u)=\lambda \mu(D+\mu) \int_{u}^{\infty} w(u, y-u) e^{-\mu y} d y .
$$

The initial value at zero $m(0)=w(0,0)$ is in this case simply the penalty function evaluated at zero. Since according to Li and Garrido (2005a), in the case of a compound Poisson risk model perturbed by a Brownian motion, the Lundberg equation has only one positive solution that we will denote $\rho$, we can apply the integrating factor technique only once. It yields the linear equation

$$
\frac{\tilde{\sigma}^{2}}{2} m^{\prime}(0)+\left(\rho \frac{\tilde{\sigma}^{2}}{2}+c\right) m(0)=\lambda \hat{\omega}(\rho)
$$

which we can solve for $m^{\prime}(0)$. With these initial values, we can compute the particular solution and Equation (31) leads to

$$
\begin{aligned}
m(u) & =-\frac{1}{\left(\rho-\sigma_{1}\right)\left(\rho-\sigma_{2}\right)} \int_{u}^{\infty} e^{\rho(u-\xi)} f(\xi) d \xi-\frac{\hat{f}(\rho)}{\sigma_{2}-\sigma_{1}}\left(\frac{e^{\sigma_{1} u}}{\rho-\sigma_{1}}-\frac{e^{\sigma_{2} u}}{\rho-\sigma_{2}}\right) \\
& +\frac{1}{\sigma_{2}-\sigma_{1}} \int_{0}^{u}\left(\frac{e^{\sigma_{1}(u-\xi)}}{\rho-\sigma_{1}}-\frac{e^{\sigma_{2}(u-\xi)}}{\rho-\sigma_{2}}\right) f(\xi) d \xi \\
& +\frac{1}{\sigma_{2}-\sigma_{1}}\left(\left[\sigma_{2} m(0)-m^{\prime}(0)\right] e^{\sigma_{1} u}+\left[-\sigma_{1} m(0)+m^{\prime}(0)\right] e^{\sigma_{2} u}\right)
\end{aligned}
$$


as an explicit expression for the Gerber-Shiu function. This formula generalizes equation (4.6) of Chen et al. (2007) for the case of exponential claim sizes and Example 1 of $\mathrm{Li}$ and Garrido (2005a) for exponential inter-arrival times.

\section{Conclusion}

We have shown that the link between symbolic computation and risk theory can be mutually fruitful and can be utilized to identify fully explicit expressions for the Gerber-Shiu function in general renewal models in terms of the employed penalty function. In the presented approach, Laplace transforms only enter in a very restricted form:

- Only the Laplace transform of the penalty (not of the Gerber-Shiu function) is computed. This has the advantage that one does not need artificial analyticity conditions on $m$.

- Moreover, the Laplace transform of the penalty is only evaluated at $\rho_{1}, \ldots, \rho_{n}$, the positive solutions of the Lundberg equation, for computing the boundary values.

- No inverse Laplace transform is involved. This is in contrast to many previous papers that give explicit formulae for the Laplace transform of the Gerber-Shiu function, which often cannot be inverted in closed form.

In principle, the symbolic method introduced in this paper can be extended to models that include investment as well as to models with interclaim time densities that satisfy ODEs with polynomial coefficients as long as the spectral structure of the Lundberg fundamental equation is still tractable. This will be pursued in future research. The factorization approach for boundary value problems generalizes in principle also to partial differential equations (Regensburger and Rosenkranz, 2009), which in the context of risk theory means that more general models including one more variable could be considered. Finally, the method may be applicable in boundary value problems that occur in other contexts in risk theory.

The formulas developed in this paper can easily be implemented in a computer algebra system, which in turn allows to quickly perform (quantitative and graphical) sensitivity analysis of the corresponding discounting penalty functions with respect to parameter and penalty changes. 


\section{Appendix: A Generalized Vandermonde Determinant}

For computing the initial values in Proposition [6] we are led to consider the $n \times n$ alternant matrix

$$
A=\left(\begin{array}{ccc}
p_{0}\left(x_{1}\right) & \cdots & p_{n-1}\left(x_{1}\right) \\
\vdots & \ddots & \vdots \\
p_{0}\left(x_{n}\right) & \cdots & p_{n-1}\left(x_{n}\right)
\end{array}\right)
$$

with polynomials $p_{i}(x)=a_{i, i} x^{i}+\ldots+a_{i, 0}$ with $a_{i, i}=1$. In the special case $p_{i}(x)=x^{i}$ this is the usual Vandermonde matrix with the determinant $V_{n}$ in the indeterminates $x_{1}, \ldots, x_{n}$, but $\operatorname{det} A=V_{n}$ holds in general (Krattenthaler, 1999, Prop. 1).

We want to compute the $(k, l)$ minor of $A$, the determinant of the $(n-$ $1) \times(n-1)$ matrix $A_{k, l}$ obtained by deleting the $k$-th row and the $l$-th column. It suffices to consider

$$
A_{n, l}=\left(\begin{array}{cccccc}
p_{0}\left(x_{1}\right) & \cdots & p_{l-1}\left(x_{1}\right) & p_{l+1}\left(x_{1}\right) & \cdots & p_{n-1}\left(x_{1}\right) \\
\vdots & \ddots & \vdots & \vdots & \ddots & \vdots \\
p_{0}\left(x_{n-1}\right) & \cdots & p_{l-1}\left(x_{n-1}\right) & p_{l+1}\left(x_{n-1}\right) & \cdots & p_{n-1}\left(x_{n-1}\right)
\end{array}\right)
$$

since

$$
A\left(x_{1}, \ldots, x_{n}\right)_{k, l}=A\left(x_{1}, \ldots, x_{k-1}, x_{k+1}, \ldots, x_{n}, x_{k}\right)_{n, l} .
$$

For $p_{i}(x)=x^{i}$ it is known (Heineman, 1929) that $\operatorname{det} A_{n, l} / V_{n-1}$ yields the elementary symmetric polynomial $e_{n-1-l}$ in $x_{1}, \ldots, x_{n-1}$.

Proposition A1. We have

$$
\frac{\operatorname{det} A_{n, l}}{V_{n-1}}=e_{n-1-l}+\sum_{j=l+1}^{n-1}\left(\sum_{J}(-1)^{j+l+m} a_{j_{1}, j_{2}} a_{j_{2}, j_{3}} \cdots a_{j_{m}, j_{m+1}}\right) e_{n-1-j},
$$

where the inner sum ranges over $J=\left(j_{1}, \ldots, j_{m+1}\right)$ such that $m \geq 1$ and $j=j_{1}>\ldots>j_{m+1}=l$.

Proof. Writing $x^{j}$ for the column vector $\left(x_{1}^{j}, \ldots, x_{n-1}^{j}\right)^{T}$, the determinant of the matrix

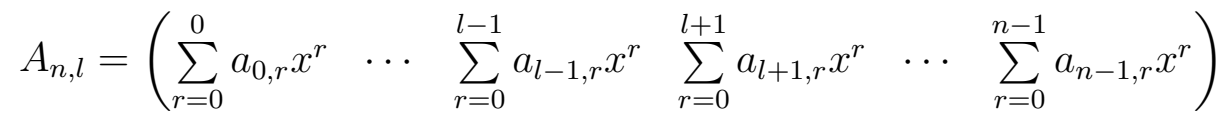

is given by multilinearity as

$$
\sum_{r_{0}=0}^{0} \cdots \sum_{r_{l-1}=0}^{l-1} \sum_{r_{l+1}=0}^{l+1} \ldots \sum_{r_{n-1}=0}^{n-1} c_{r} \operatorname{det}\left(x^{r_{0}}, \ldots, x^{r_{l-1}}, x^{r_{l+1}}, \ldots, x^{r_{n-1}}\right)
$$


with $c_{r}=a_{0, r_{0}} \cdots a_{l-1, r_{l-1}} a_{l+1, r_{l+1}} \cdots a_{n-1, r_{n-1}}$. Observe that for the first $l$ indices $r_{0}, \ldots, r_{l-1}$ there always exist some $i<j<l$ such that $r_{i}=r_{j}$ unless $r_{i}=i$ for all $i<l$. Since the determinant vanishes for the cases $r_{i}=r_{j}$ and the $p_{i}$ are monic (i.e., $a_{i, i}=1$ ), the determinant reduces to

$$
\sum_{r_{l+1}=l}^{l+1} \cdots \sum_{r_{n-1}=l}^{n-1} a_{l+1, r_{l+1}} \cdots a_{n-1, r_{n-1}} \operatorname{det}\left(x^{0}, \ldots, x^{l-1}, x^{r_{l+1}}, \ldots, x^{r_{n-1}}\right)
$$

where $r_{l+1}, \ldots, r_{n-1}$ can be restricted to mutually distinct indices.

We view the indices as the permutations $r:\{l, \ldots, n-1\} \rightarrow\{l, \ldots, n-1\}$ satisfying $r_{s} \leq s$ for $s>l$; note that $r_{l}$ is determined as the index omitted in $r_{l+1}, \ldots, r_{n-1}$. By the monotonicity condition on $r$, all cycles without $l$ in the cycle representation of $r$ are trivial: If we have a nontrivial cycle $\left(j_{1} \ldots j_{m+1}\right)$, with $j_{m+1} \neq l$ we are led to the contradiction $j_{1}>r_{j_{1}}=j_{2}>$ $\ldots>j_{m+1}>r_{j_{m+1}}=j_{1}$. Consequently $r$ either possesses only one nontrivial cycle $\left(j_{1} \ldots j_{m+1}\right)$ with $j_{1}>\ldots>j_{m+1}=l$, unless $r$ is the identity. Since the $p_{i}$ are monic, the factor of the determinant $\Delta_{r}$ occurring in the above sum is given by $a_{j_{1}, j_{2}} \cdots a_{j_{m}, j_{m+1}}$ in the former and by 1 in the latter case.

For finding $\Delta_{r}$, we use row expansion for computing

$$
(-1)^{l+n-1} \Delta_{r}=\operatorname{det}\left(\begin{array}{ccccccc}
x^{0} & \cdots & x^{l-1} & x^{l} & x^{r_{l+1}} & \cdots & x^{r_{n-1}} \\
0 & \cdots & 0 & 1 & 0 & \cdots & 0
\end{array}\right) .
$$

This determinant is the result of $r$ acting on the columns of the determinant

$$
\operatorname{det}\left(\begin{array}{ccccccc}
x^{0} & \cdots & x^{j-1} & x^{j} & x^{j+1} & \cdots & x^{n-1} \\
0 & \cdots & 0 & 1 & 0 & \cdots & 0
\end{array}\right)=(-1)^{j+n-1} e_{n-1-j} V_{n-1}
$$

according to the above mentioned result on Vandermonde minors. Since $r$ as a cycle of length $m+1$ has sign $(-1)^{m}$, this yields

$$
\Delta_{r}=(-1)^{j+l+m} e_{n-1-j} V_{n-1},
$$

which proves the formula.

Note that the inner sum in Proposition A1 can also be interpreted as ranging over all ordered subsets of $\{l, \ldots, j\}$ containing $l$ and $j$. It can be simplified further in the following special case, which we use in Section 6. We give two representations, one in terms of compositions and the other using generating functions. Here we use the customary notation $\left[x^{i}\right] f(x)$ for the coefficient of $x^{i}$ in a power series $f(x)$. 
Corollary A2. If $p_{i}(x)=a_{0} x^{i}+\ldots+a_{i}, a_{0}=1$, the formula in Proposition A1 simplifies to

$$
\frac{\operatorname{det} A_{n, l}}{V_{n-1}}=e_{n-1-l}+\sum_{j=l+1}^{n-1}(-1)^{j-l}\left(\sum_{m \geq 1}(-1)^{m} \sum_{d_{1}, \ldots, d_{m}} a_{d_{1}} \ldots a_{d_{m}}\right) e_{n-1-j},
$$

where the inner sum ranges over $d_{1}, \ldots, d_{m}>0$ such that $d_{1}+\ldots+d_{m}=j-l$. Using generating functions, we have also

$$
\frac{\operatorname{det} A_{n, l}}{V_{n-1}}=\sum_{j=0}^{n-1-l}\left(\left[x^{j}\right] \frac{(-1)^{j}+q(x)^{j+1}}{1+q(x)}\right) e_{n-1-l-j},
$$

where $q(x)=a_{1} x+\ldots+a_{n-1} x^{n-1}$.

Proof. Applying the above remark to the case $a_{i, j}=a_{i-j}$, the inner sum in Proposition A1 gives

$$
\begin{aligned}
\sum_{j>j_{2}>\cdots>j_{m}>l}(-1)^{m} a_{j-j_{2}} a_{j_{2}-j_{3}} \cdots a_{j_{m-1}-j_{m}} a_{j_{m}-l} \\
=\sum_{d_{1}, \ldots, d_{m}>0, \sum_{i} d_{i}=j-l}(-1)^{m} a_{d_{1}} \ldots a_{d_{m}}
\end{aligned}
$$

for $j>l$, since the differences $d_{1}=j-j_{2}, d_{2}=j_{2}-j_{3}, \ldots, d_{m}=j_{m}-l$ can take arbitrary nonnegative values, provided they sum up to $j-l$. Now the first formula follows by multiplying with $(-1)^{j+l}=(-1)^{j-l}$.

For the second formula observe that the sum over the compositions of $j-l$ that appears within the bracket of the first formula is equal to the coefficient of $x^{j-l}$ in the product

$$
\prod_{i=1}^{m}\left(a_{1} x+\ldots+a_{n-1} x^{n-1}\right)=q(x)^{m},
$$

for $m \leq j-l$; for $m>j-l$ the sum over the composition is empty. Note that this even covers the cases $m=0$, for which the term is zero except for $j-l=0$, when it becomes one. The stated formula then follows by

$$
\begin{array}{r}
(-1)^{j-l} \sum_{m=0}^{j-l}\left[x^{j-l}\right](-q(x))^{m}=(-1)^{j-l}\left[x^{j-l}\right] \frac{1-(-q(x))^{j-l+1}}{1+q(x)} \\
=\left[x^{j-l}\right] \frac{(-1)^{j-l}+q(x)^{j-l+1}}{1+q(x)} .
\end{array}
$$


As a final remark note that the determinant takes an even simpler form if the $p_{i}$ are not 'reversed' as they are in the previous corollary.

Corollary A3. If $p_{i}(x)=a_{i} x^{i}+\ldots+a_{0}$, the formula in Proposition A1 simplifies to

$$
\frac{\operatorname{det} A_{n, l}}{V_{n-1}}=e_{n-1-l}+\sum_{j=l+1}^{n-1}\left(a_{l} \prod_{k=l+1}^{j-1}\left(a_{k}-1\right)\right) e_{n-1-j}
$$

Proof. The proof proceeds in a similar way as for the previous corollary. Here we have the case $a_{i, j}=a_{j}$, so the inner sum in Proposition A1 evaluates to

$$
\sum_{\substack{\left\{j_{2}, \ldots, j_{m}\right\} \subseteq\{l+1, \ldots, j-1\} \\ j>j_{2}>\cdots>j_{m}>l}}(-1)^{m} a_{j_{2}} \cdots a_{j_{m}} a_{j_{m+1}}=a_{l} \prod_{k=l+1}^{j-1}\left(a_{k}-1\right),
$$

and the rest follows.

\section{Acknowledgements}

We would like to thank Jose Garrido for his encouraging feedback at an early stage of this project. We would also like to thank Christian Krattenthaler for valuable hints on simplifying the determinant of Proposition A1 and Peter Paule and Christoph Koutschan for help with proving the explicit form of $d_{j}$ in the proof of Proposition 6. Furthermore we thank the anonymous referees for helpful comments to improve the presentation of the manuscript. Hansjörg Albrecher was partly supported by the Austrian Science Fund Project P18392, Gottlieb Pirsic was partly supported by the Austrian Science Fund Project P19004-N18.

\section{References}

Albrecher, H., Boxma, O. J., 2005. On the discounted penalty function in a Markov-dependent risk model. Insurance: Mathematics \& Economics 37 (3), 650-672.

Bingham, N. H., Goldie, C. M., Teugels, J. L., 1987. Regular Variation. Vol. 27 of Encyclopedia of Mathematics and its Applications. Cambridge: Cambridge University Press.

Bronstein, M., 2005. Symbolic Integration. I, 2nd Edition. Vol. 1 of Algorithms and Computation in Mathematics. Berlin: Springer-Verlag. 
Butzer, P. L., Berens, H., 1967. Semi-groups of Operators and Approximation. Die Grundlehren der mathematischen Wissenschaften, Band 145. New York: Springer-Verlag New York Inc.

Chen, Y.-T., Lee, C.-F., Sheu, Y.-C., 2007. An ODE approach for the expected discounted penalty at ruin in a jump-diffusion model. Finance and Stochastics 11 (3), 323-355.

Cheng, Y., Tang, Q., 2003. Moments of the surplus before ruin and the deficit at ruin in the Erlang(2) risk process. North American Actuarial Journal $7(1), 1-12$.

Constantinescu, C., 2006. Renewal Risk Processes with Stochastic Returns on Investments - A Unified Approach and Analysis of the Ruin Probabilities, PhD Thesis Edition. Corvallis, OR: Valley Library.

Conway, J. B., 1990. A Course in Functional Analysis, 2nd Edition. Vol. 96 of Graduate Texts in Mathematics. New York: Springer-Verlag.

Dickson, D. C. M., Hipp, C., 2001. On the time to ruin for Erlang(2) risk processes. Insurance: Mathematics \& Economics 29 (3), 333-344.

Drekic, S., Stafford, J. E., Willmot, G. E., 2004. Symbolic calculation of the moments of the time of ruin. Insurance: Mathematics \& Economics 34 (1), $109-120$.

Feller, W., 1971. An Introduction to Probability Theory and its Applications. Vol. II. Second edition. New York: John Wiley \& Sons Inc.

Gerber, H. U., Shiu, E. S. W., 1997. The joint distribution of the time of ruin, the surplus immediately before ruin, and the deficit at ruin. Insurance: Mathematics \& Economics 21 (2), 129-137.

Gerber, H. U., Shiu, E. S. W., 1998. On the time value of ruin. North American Actuarial Journal 2 (1), 48-78.

Gerber, H. U., Shiu, E. S. W., 2005. The time value of ruin in a Sparre Andersen model. North American Actuarial Journal 9 (2), 49-84.

Graham, R. L., Knuth, D. E., Patashnik, O., 1989. Concrete Mathematics. Reading, MA: Addison-Wesley Publishing Company Advanced Book Program.

Heineman, E. R., 1929. Generalized Vandermonde determinants. Transactions of the American Mathematical Society 31 (3), 464-476. 
Kamke, E., 1967. Differentialgleichungen. Lösungsmethoden und Lösungen. Teil I: Gewöhnliche Differentialgleichungen, eighth Edition. Vol. 18 of Mathematik und ihre Anwendungen in Physik und Technik A. Leipzig: Akademische Verlagsgesellschaft.

Krattenthaler, C., 1999. Advanced determinant calculus. Séminaire Lotharingien de Combinatoire 42, Art. B42q, 67 pp. (electronic), the Andrews Festschrift (Maratea, 1998).

Landriault, D., Willmot, G., 2008. On the Gerber-Shiu discounted penalty function in the Sparre Andersen model with an arbitrary interclaim time distribution. Insurance: Mathematics \& Economics 42 (2), 600-608.

Li, S., Garrido, J., 2004. On ruin for the $\operatorname{Erlang}(n)$ risk process. Insurance: Mathematics \& Economics 34 (3), 391-408.

Li, S., Garrido, J., 2005a. The Gerber-Shiu function in a Sparre Andersen risk process perturbed by diffusion. Scandinavian Actuarial Journal (3), $161-186$.

Li, S., Garrido, J., 2005b. On a general class of renewal risk process: analysis of the Gerber-Shiu function. Advances in Applied Probability 37 (3), 836856.

Lin, X. S., Willmot, G. E., 2000. The moments of the time of ruin, the surplus before ruin, and the deficit at ruin. Insurance: Mathematics \& Economics 27 (1), 19-44.

Regensburger, G., Rosenkranz, M., 2009. An algebraic foundation for factoring linear boundary problems. Annali di Matematica Pura ed Applicata. Series IV 188 (1), 123-151.

Rosenkranz, M., 2005. A new symbolic method for solving linear two-point boundary value problems on the level of operators. Journal of Symbolic Computation 39 (2), 171-199.

Rosenkranz, M., Regensburger, G., 2008. Solving and factoring boundary problems for linear ordinary differential equations in differential algebras. Journal of Symbolic Computation 43 (8), 515-544.

Sparre Andersen, E., 1957. On the collective theory of risk in case of contagion between claims. Bulletin of the Institute of Mathematics and its Applications 12, 275-279. 
Stakgold, I., 2000. Boundary Value Problems of Mathematical Physics. Vol. I, II. Vol. 29 of Classics in Applied Mathematics. Philadelphia, PA: Society for Industrial and Applied Mathematics (SIAM), corrected reprint of the 1967-68 original.

Stanley, R. P., 1999. Enumerative Combinatorics. Vol. 2. Vol. 62 of Cambridge Studies in Advanced Mathematics. Cambridge: Cambridge University Press.

Willmot, G. E., 1999. A Laplace transform representation in a class of renewal queueing and risk processes. Journal of Applied Probability 36 (2), 570584.

Willmot, G. E., 2007. On the discounted penalty function in the renewal risk model with general interclaim times. Insurance: Mathematics \& Economics 41 (1), 17-31.

Yosida, K., 1995. Functional Analysis. Classics in Mathematics. Berlin: Springer-Verlag, reprint of the sixth (1980) edition. 